\title{
Effect of perturbations on the non linear stability of triangular points in the restricted three-body problem with variable mass
}

\author{
Jagadish Singh
}

Received: 19 November 2008 / Accepted: 23 February 2009 / Published online: 27 March 2009

(C) The Author(s) 2009. This article is published with open access at Springerlink.com

\begin{abstract}
The effect of small perturbations $\varepsilon$ and $\varepsilon^{\prime}$ in the Coriolis and the centrifugal forces, respectively on the nonlinear stability of the triangular points in the restricted threebody problem with variable mass has been studied. It is found that, in the nonlinear sense, the triangular points are stable for all mass ratios in the range of linear stability except for three mass ratios, which depend upon $\varepsilon, \varepsilon^{\prime}$ and $\beta$, the constant due to the variation in mass governed by Jeans' law.
\end{abstract}

Keywords Perturbations · Nonlinear stability · Triangular points $\cdot$ RTBP with variable mass

\section{Introduction}

The restricted three-body problem describes the motion of an infinitesimal mass moving in the gravitational field of two massive bodies, called primaries, which move in circular Keplerian orbits about their common center of mass. The infinitesimal mass can be at rest in a rotating coordinate frame, at five equilibrium points, where the gravitational and centrifugal forces just balance each other. Three of them are the collinear points $\mathrm{L}_{1}, \mathrm{~L}_{2}, \mathrm{~L}_{3}$ aligned with the two primaries, while the last two are linearly stable if the mass ratio of the primaries is less than 0.03852 ... (Szebehely 1967a). Their stability occurs in spite of the fact that the potential energy has a maximum rather than a minimum at $\mathrm{L}_{4}$ and $\mathrm{L}_{5}$. The stability is actually achieved through the influence

J. Singh ( $\varangle)$

Department of Mathematics, Faculty of science, Ahmadu Bello

University (ABU), Zaria, Nigeria

e-mail: jgds2004@yahoo.com of the Coriolis force, because the coordinate system is rotating (Wintner 1941; Contopoulos 2002).

In this classical formulation of the problem, the effects of the gravitational attraction of the infinitesimal body and other perturbations have been ignored. Perturbations can well arise from the causes such as the lack of sphericity, or the oblateness and the radiation forces of the bodies, variation of the masses, the atmospheric drag, the solar wind, the Poynting Robertson effect and the action of other bodies. The Kirkwood gaps in the ring of the asteroid's orbits lying between the orbits of the Mars and Jupiter are the examples of the perturbation produced by the Jupiter on an asteroid. Another example is the motion of a close artificial satellite of the Earth perturbed by the atmospheric friction and the oblateness of the Earth. This enables many researchers to study the restricted problem by taking into account the effect of small perturbations in the Coriolis and the centrifugal forces, radiation and the oblateness of the bodies (Szebehely 1967b; Subbarao and Sharma 1975; AbdulRaheem and Singh 2006).

For the stability of the triangular points Szebehely (1967b) asserted the Coriolis force is a stabilizing force, whereas Subbarao and Sharma (1975) observed that the oblateness of the primary resulted in an increase in both the Coriolis and the centrifugal forces, thereby concluding that the Coriolis force is not always a stabilizing force. The stability of the triangular points under the influence of small perturbations in the Coriolis and the centrifugal forces together with the effect of oblateness and radiation pressures of the primaries was investigated by AbdulRaheem and Singh (2006). It was found that the Coriolis has a stabilizing tendency, while the centrifugal force, radiation, and oblateness of the primaries have destabilizing effects; the presence of any one or more of the latter makes weak the 
stabilizing ability of the former. The overall effect is that the range of its stability decreases.

Modern concepts about the formulation of celestial bodies under their evolution lead to the necessity of investigating dynamical problems in celestial mechanics in which the motion of the bodies is determined by nonsteady schemes. The masses of celestial bodies are changing during evolution. The isotropic variation of the masses of the bodies occurs due to radiation or absorption of light. As an example of isotropic mass variation we can point to the decrease in stellar mass by light emission or corpuscularly. A satellite moving around a radiating star surrounded by cloud variates its mass due to particles of this cloud. The two-body problem with variable mass came into science practically following the work of Jeans (1928) in the study of the evolution of a binary system. Since then, many important results have been obtained from the physical and the mathematical point of view. Meshcherskii (1949) assumed that mass is ejected isotropically from the two-body system at very high velocity and is lost to the system. Verhulst (1972) described the two-body problem with slowly decreasing mass according to Jean's (1928) law.

The bodies in the classical restricted three-body problem are strictly of constant mass, but there are numerous practical problems where the mass does not remain constant. It has been shown that the mass of Jupiter is increasing (Shrivastava and Ishwar 1983). The phenomenon of isotropic radiation or absorption of mass in stars leads to the concept of the decreasing mass. Also, the classical model assumes that the third body of infinitesimal mass moves under only the mutual gravitational force of the primaries, but in practice, due to rotational motions, Coriolis and centrifugal forces are effective and small perturbations affect these forces. Thus, the above considerations concerned to the classical problem are not justified, and the results obtained are far from a realistic approach.

The effect of small perturbations $\varepsilon$ and $\varepsilon^{\prime}$ in the Coriolis and the centrifugal forces on the location and the linear stability of equilibrium points in the restricted problem of three bodies with variable (decreasing) mass was analyzed by the authors (Singh and Ishwar 1984, 1985) under the assumption that the variation in mass of the third body is governed by Jeans' (1928) law. It may be here noted that Jeans' (1928) law is applicable in the case when only one body decreases its mass isotropically, which is the case under consideration. The authors found that triangular points form nearly equilateral triangles with the primaries and they are stable for $0<\mu<\mu_{\mathrm{c}}$ and unstable for $\mu_{\mathrm{c}}<\mu<\frac{1}{2}$, where $\mu_{\mathrm{c}}$ is a critical value of the mass parameter.

Many Mathematicians and astronomers have been interested in the study of stability of an equilibrium point for all time and all the orders of the terms in the expansion of the Lagrangian or Hamiltonian. The nonlinear stability of triangular points in the classical restricted three-body problem was investigated by Deprit and Deprit Bartholome (1967). Bhatnagar and Hallan (1978) discussed the effects of perturbations in the Coriolis and centrifugal forces, respectively on the nonlinear stability of equilibrium points of the same problem. Later, analytical studies $f$ the nonlinear stability of $\mathrm{L}_{4,5}$ under various aspects were also carried out by Niedzielska (1994), Ishwar (1997), Subbarao and Sharma (1997), Hallan et al. (2000) and Gozdziewski (2003). In this paper, we propose to study the effect of small perturbations in the Coriolis and centrifugal forces on the nonlinear stability of the triangular points in the restricted three-body problem with variable (decreasing) mass under the assumption that the mass of the third body varies with time.

Our aim is to consider variable mass together with small perturbations in the Coriolis and centrifugal forces as it has been found that they produce significant changes in the location (Singh and Ishwar 1984) and the stability (Singh and Ishwar 1985) of the equilibrium points. We also know that the inclusion of the nonlinear terms sometimes changes the entire pattern of the stability and hence, we intend to studying the stability in the non-linear sense as well. The masses of celestial bodies are changing during evolution, therefore the restricted problem with variable mass may be used in different astronomical problems (Bekov 1992). Thus, it has theoretical and practical importance. The proposed model is best suited to some of the well known stellar systems such as the sun-earth-star system. This model is more realistic than the classical restricted problem with reference to it's application in the stellar systems.

By applying Liapunov's (1956) theorem to the linear stability results in Singh and Ishwar (1985) mentioned earlier, we can say that the triangular points for $\mu_{\mathrm{c}}<\mu<\frac{1}{2}$ are unstable in the nonlinear sense also. So, we need to study the nonlinear stability of triangular points for $0<\mu<\mu_{\mathrm{c}}$. For this, we will apply Moser's (1962) modified version of Arnold's (1961) theorem and follow the method as adopted by Bhatnagar and Hallan (1978). Arnold's (1961) proved that if

i. $k_{1} \omega_{1}+k_{2} \omega_{2} \neq 0$ for all pairs $\left(k_{1}, k_{2}\right)$ of rational integers and

ii. the determinant $D \neq 0$, where $\omega_{1}, \omega_{2}$ are basic frequencies for the linear dynamical system, $D=\operatorname{det}\left(b_{i j}\right)$, $(i, j=1,2,3)$,

$$
\begin{aligned}
& b_{i j}=\left(\frac{\partial^{2} \mathrm{H}}{\partial I_{i} d I_{j}}\right) I_{i}=I_{j}=0 \quad(i, j=1,2), \\
& b_{i 3}=b_{3 i}=\left(\frac{\partial \mathrm{H}}{\partial I_{i}}\right) I_{i}=I_{j}=0 \quad(i=1,2), b_{33}=0
\end{aligned}
$$

and

$\mathrm{H}=\omega_{1} I_{1}-\omega_{2} I_{2}+\frac{1}{2}\left(A I_{1}^{2}+2 \bar{B} I_{1} I_{2}+C I_{2}^{2}+\cdots\right)$ 
is the normalized Hamiltonian with $I_{1}, I_{2}$ as the action momenta coordinates, and $A, \bar{B}, C$ as second order coefficients in the frequencies, then on each energy manifold $\mathrm{H}=h$ in the neighbourhood of equilibrium, there exist in variant tori of quasi-periodic motions which divide the manifolds, and consequently, the equilibrium is stable. This is valid for a system with two degrees of freedom, which is the case under consideration. Moser (1962) showed that Arnold's (1961) theorem is true if the condition (i) of the theorem is replaced by

$k_{1} \omega_{1}+k_{2} \omega_{2} \neq 0 \quad$ for all pairs $\left(k_{1}, k_{2}\right)$ of rational integers such that $\left|k_{1}\right|+\left|k_{2}\right| \leq 4$

This paper should be read in conjunction with the paper by Bhatnagar and Hallan (1978) as, to save space, we are not mentioning the values of various variables given in that paper, although they are used in this paper.

\section{Equations of motion}

The equations of motion of the infinitesimal variable (decreasing) mass in the restricted three-body problem in a synodic coordinate system are given by Singh and Ishwar (1984) as:

$\xi^{\prime \prime}-2 \phi \eta^{\prime}=\frac{\partial \Omega}{\partial \xi}, \quad \eta^{\prime \prime}+2 \phi \xi^{\prime}=\frac{\partial \Omega}{\partial \eta}$

where $\Omega=\frac{B}{2} \Psi\left(\xi^{2}+\eta^{2}\right)+\gamma^{3 / 2}\left(\frac{1-\mu}{r_{1}}+\frac{\mu}{r_{2}}\right)$.

$r_{1}^{2}=\left(\xi+\mu \gamma^{1 / 2}\right)^{2}+\eta^{2}, \quad r_{2}^{2}=\left\{\xi-(1-\mu) \gamma^{1 / 2}\right\}^{2}+\eta^{2}$

$B=\frac{\beta^{2}}{4}+1, \quad \gamma=\frac{m}{m_{0}}, \quad \beta=\alpha m_{0}^{n-1}$

where $\alpha$ is a constant coefficient governed by Jeans' (1928) law $\dot{m}=-\alpha m^{n}$ and the value of exponent $n$ is within the limit $0.4 \leq n \leq 4.4$ for the star of the main sequence. The parameter $\mu$ is the ratio of the mass of the smaller primary to the total mass of the primaries and $0<\mu \leq \frac{1}{2}$. The mass $m$ of the third body varies with the time $t$ and it becomes $m_{0}$ when $t=0$. Primes indicate differentiation with respect to $\Gamma$ where $d t=\gamma^{-k} d \Gamma, k$ is a constant of proportionality. Perturbations in the Coriolis and the centrifugal forces are introduced with the help of the parameters $\phi$ and $\Psi$. The unperturbed value of each is unity.

\section{Triangular equilibrium points}

The locations $(\xi, \pm \eta)$ of the triangular points $\mathrm{L}_{4}$ and $\mathrm{L}_{5}$ can be written as (Singh and Ishwar 1984).

$\xi=\left(\frac{1}{2}-\mu\right) \gamma^{1 / 2}, \quad \eta=\frac{\gamma^{1 / 2}}{2}\left[4(B \Psi)^{-2 / 3}-1\right]^{1 / 2}$

\section{Linear stability}

Since the nature of the stability about the $\mathrm{L}_{5}$ point will be similar to that about $\mathrm{L}_{4}$, it will be sufficient to consider here the stability only near $\mathrm{L}_{4}$. The system (1) describes the motion of a dynamical system with Lagrangian given by

$$
\begin{aligned}
L= & \frac{1}{2}\left(\xi^{\prime 2}+\eta^{\prime 2}\right)+\phi\left(\xi \eta^{\prime}-\eta \xi^{\prime}\right) \\
& +\frac{B \varphi}{2}\left(\xi^{2}+\eta^{2}\right)+\gamma^{3 / 2}\left(\frac{1-\mu}{r_{1}}+\frac{\mu}{r_{2}}\right)
\end{aligned}
$$

For simplicity $\phi$ and $\Psi$ may be taken as

$$
\phi=1+\varepsilon, \quad|\varepsilon| \ll 1, \quad \Psi=1+\varepsilon^{\prime}, \quad\left|\varepsilon^{\prime}\right| \gg 1
$$

where $\varepsilon, \varepsilon^{\prime}$ represent the perturbations in the Coriolis and the centrifugal forces respectively. In the computation, we will ignore the second and higher order terms in $\varepsilon, \varepsilon^{\prime}$ and their product with $\beta^{2}$, where $\beta^{2}$ is very small.

Shifting the origin to $\mathrm{L}_{4}$ and expanding in power series of $\xi$ and $\eta$, we have

$L=\mathrm{L}_{0}+\mathrm{L}_{1}+\mathrm{L}_{2}+\mathrm{L}_{3}+\mathrm{L}_{4}+$

where

$$
\begin{aligned}
\mathrm{L}_{0}= & \frac{1}{8} \gamma B\left[s^{2}-1+12 B^{-\frac{2}{3}}+\left(s^{2}+3\right) \varepsilon^{\prime}\right], \\
\mathrm{L}_{1}= & \frac{1}{2} \gamma^{1 / 2}(1+\varepsilon)\left(s \eta^{\prime}-d \xi^{\prime}\right), \\
\mathrm{L}_{2}= & \frac{1}{2}\left(\xi^{\prime 2}+\eta^{\prime 2}\right)+\left(\xi \eta^{\prime}-\xi^{\prime} \eta\right)(1+\varepsilon)+\frac{1}{8} B^{5 / 3} b \xi^{2} \\
& +\frac{1}{8} B^{5 / 3}\left[12 B^{-2 / 3}\left(1+\varepsilon^{\prime}\right)-b\right] \eta^{2}+\frac{1}{4} B^{5 / 3} b d s \xi \eta, \\
\mathrm{L}_{3}= & \frac{1}{48} s \gamma^{-1 / 2}\left(12 b-5 B^{\frac{2}{3}} C\right) B^{5 / 3} \xi^{3} \\
& -\frac{1}{16} \gamma^{-\frac{1}{2}} B^{\frac{5}{3}}\left(5 B^{\frac{2}{3}} C-4 b\right) d \xi^{2} \eta-\frac{1}{16} \gamma^{-\frac{1}{2}} s B^{\frac{5}{3}}, \\
& \left(16 b-5 B^{\frac{2}{3}} C\right) \xi \eta^{2}-\frac{1}{48} \gamma^{-\frac{1}{2}} B^{\frac{5}{3}}\left(8 b-5 B^{\frac{2}{3}} C\right) d \eta^{3}, \\
\mathrm{~L}_{4}= & -\frac{1}{128} \gamma^{-1}\left(40 B^{\frac{5}{3}} C-35 a B^{\frac{4}{3}}-16 b\right) \xi^{4} \\
& -\frac{5 \gamma^{-1} s}{32} B^{\frac{7}{3}}\left(4 C-7 a B^{\frac{2}{3}}\right) d \xi^{3} \eta+\frac{1}{64} \gamma^{-1} B^{\frac{5}{3}} \\
& \times\left(140 C B^{\frac{2}{3}}-64 b-105 a B^{\frac{4}{3}}\right) \xi^{2} \eta^{2} \\
& +\frac{5}{96} \gamma^{-1} S B^{\frac{7}{3}}\left(16 C-21 a B^{\frac{2}{3}}\right) d \xi \eta^{3} \\
& +\frac{1}{384} \gamma^{-1} B^{\frac{5}{3}}\left(128 b-160 C B^{\frac{2}{3}}+105 a\right) \eta^{4}, \\
a=1+ & 3 \varepsilon^{\prime}, \quad b=3+5 \varepsilon^{\prime}, \quad c=3+7 \varepsilon^{\prime},
\end{aligned}
$$


$d=\left\{4 B^{-2 / 3}\left(1-\frac{2}{3} \varepsilon^{\prime}\right)-1\right\}^{\frac{1}{2}}, \quad s=1-2 \mu$

Here $\mathrm{L}_{4}$ denotes the fourth order part of the Lagrangian.

To the first order, Lagrange's equations of motion can be written as

$\xi^{\prime \prime}-2(1+\varepsilon) \eta^{\prime}=\frac{1}{4} B^{\frac{5}{3}} b(\xi+d) s \eta$,

$\eta^{\prime \prime}+2(1+\varepsilon) \xi^{\prime}=\frac{1}{4} B^{\frac{5}{3}}\left[b d s \xi+\left(12 B^{-\frac{2}{3}}\left(1+\varepsilon^{\prime}\right)-b\right) \eta\right]$

The characteristics equation of these equations is given by

$$
\begin{aligned}
\lambda^{4}+ & {\left[1+8 \varepsilon-3 \varepsilon^{\prime}+3(1-B)\left(1+\varepsilon^{\prime}\right)\right] \lambda^{2}+\frac{3}{16}\left(1-s^{2}\right) } \\
\times & B^{\frac{10}{3}}\left(4 B^{-\frac{2}{3}}\left(3+8 \varepsilon^{\prime}\right)-\left(3+10 \varepsilon^{\prime}\right)\right)=0
\end{aligned}
$$

The roots of (6) are purely imaginary if its discriminant

$$
\begin{aligned}
\Delta= & \left(4-3 B+8 \varepsilon-3 B \varepsilon^{1}\right)^{2}-\frac{3}{4}\left(1-s^{2}\right) \\
& \times B^{\frac{10}{3}}\left[3\left(4 B^{-\frac{2}{3}}-1\right)+\left(32 B^{-2 / 3}-10\right) \varepsilon^{\prime}\right]>0
\end{aligned}
$$

and thus it is a necessary condition for the stability, in the linear sense, around the $\mathrm{L}_{4}$ point.

The solution of the quadratic equation $\Delta=0$ for $\mu$ gives the critical mass value $\mu_{\mathrm{c}}$ of the mass parameter. That is

$\mu_{c}=\frac{1}{2}-\frac{1}{2}\left[1-\frac{4(4-3 B)^{2}}{9 B^{\frac{10}{3}}\left(4 B^{-\frac{2}{3}}-1\right)}\right]^{\frac{1}{2}}+\frac{4\left(36 \varepsilon-19 \varepsilon^{\prime}\right)}{27 \sqrt{69}}$

Hence the range of stability, in the linear sense, can be written as $0<\mu<\mu_{\mathrm{c}}$.

If (6) has four imaginary roots $\pm i \omega_{1}^{\prime}, \pm i \omega_{2}^{\prime}$, where $\omega_{1}^{\prime}$, $\omega_{2}^{\prime}$ are the perturbed basic frequencies, then we have

$\omega_{1}^{\prime}=\omega_{1}\left(1+P \varepsilon+P^{\prime} \varepsilon^{\prime}\right)$ and $\omega_{2}^{\prime}=\omega_{2}\left(1+q \varepsilon+q^{\prime} \varepsilon^{\prime}\right)$

where $\omega_{1}, \omega_{2}$ represent the unperturbed basic frequencies such that

$\omega_{1}^{2}+\omega_{2}^{2}=4-3 B, \quad \omega_{1}^{2} \omega_{2}^{2}=\frac{9}{16}\left(1-s^{2}\right) B^{\frac{10}{3}}\left(4 B^{-\frac{2}{3}}-1\right)$,

$0<\omega_{2}<\left(\frac{4-3 B}{2}\right)^{\frac{1}{2}}<\omega_{1}<(4-3 B)^{\frac{1}{2}}$

and $p=-q=\frac{4}{k^{2}}, k^{2}=\omega_{1}^{2}-\omega_{2}^{2}=2 \omega_{1}^{2}-1+\frac{3}{4} \beta^{2}=1-$ $2 \omega_{2}^{2}-\frac{3}{4} \beta_{0}^{2}$,

$p^{\prime}=\frac{22 \omega_{1}^{2}-49}{18 k^{2}}, \quad q^{\prime}=-\frac{22 \omega_{2}^{2}-49}{18 k^{2}}$

Here only powers up to second order of $\beta$ are retained as it is very small. The products $\beta^{2} \varepsilon$ and $\beta^{2}+\frac{1}{2}\left[1+2 \varepsilon+\frac{3}{4} B^{\frac{5}{3}}(1+\right.$ $\left.\left.\frac{5}{3} \xi^{\prime}\right)-3 B\left(1+\varepsilon^{\prime}\right)\right] \eta^{2}-\frac{3}{4} s B^{\frac{5}{3}}\left(1+\frac{5}{3} \varepsilon^{\prime}\right)\left[4 B^{-\frac{2}{3}}\left(1-\frac{2}{3} \varepsilon^{\prime}\right)-\right.$ $1]^{\frac{1}{2}} \xi \eta$ are also neglected. One can see that the values of $p$, $p^{\prime}, q, q^{\prime}$ agree with those of Bhatnagar and Hallan (1978).

The second order part of the Hamiltonian is given by

$$
\begin{aligned}
\mathrm{H}_{2}= & \frac{1}{2}\left(P_{\xi}^{2}+P_{\eta}^{2}\right)+\left(\eta P_{\xi}-\xi P_{\eta}\right)(1+\varepsilon) \\
& +\frac{1}{2}\left[1+2 \varepsilon-\frac{3}{4} B^{\frac{5}{3}}\left(1+\frac{5}{3} \varepsilon^{\prime}\right)\right] \xi^{2} \\
& +\frac{1}{2}\left[1+2 \varepsilon+\frac{3}{4} B^{\frac{5}{3}}\left(1+\frac{5}{3} \xi^{\prime}\right)-3 B\left(1+\varepsilon^{\prime}\right)\right] \eta^{2} \\
& -\frac{3}{4} s B^{\frac{5}{3}}\left(1+\frac{5}{3} \varepsilon^{\prime}\right)\left[4 B^{-\frac{2}{3}}\left(1-\frac{2}{3} \varepsilon^{\prime}\right)-1\right]^{\frac{1}{2}} \xi \eta
\end{aligned}
$$

If $\mathrm{H}_{2}$ is of positive definite form, then the equilibrium solution is stable by virtue of Liapunov's (1956) theorem for all orders and all time. On the other hand, if $\mathrm{H}_{2}$ is not a function of definite sign, then the stability can be investigated by means of KAM theorem. However, as is clear from (10) the function $\mathrm{H}_{2}$ is not sign definite and hence we can not draw any conclusion about the stability of the whole system. To make use of KAM theorem it is necessary to reduce the Hamiltonian to its normalized form. So, we perform the first and second order normalization. The first order normalization is to transform the square part of the Hamiltonian to the form corresponding to normal oscillations. In the computation of the second order coefficients in the frequencies, the coefficients of sine and cosine occur as critical terms. These critical terms are eliminated by choosing properly the coefficients in the polynomials.

\section{First order normalization}

In order to perform the first order normalization we apply the canonical transformation from the phase space $\left(\xi, \eta, P_{\xi}, P_{\eta}\right)$ into the phase space product of the angle coordinates $\left(\theta_{1}, \theta_{2}\right)$ and the action momenta $\left(I_{1}, I_{2}\right)$ as in Whittaker (1965).

$\left[\begin{array}{c}\xi \\ \eta \\ P_{\xi} \\ P_{\eta}\end{array}\right]=A^{\prime}\left[\begin{array}{c}Q_{1} \\ Q_{2} \\ P_{1} \\ P_{2}\end{array}\right]$

where

$$
\begin{aligned}
& A^{\prime}=\left(a_{i j}^{\prime}\right) \quad 1 \leq i, j \leq 4, \quad Q_{\mathrm{i}}=\left(\frac{2 I_{i}}{\omega_{i}^{\prime}}\right)^{\frac{1}{2}} \sin \theta_{i}, \\
& P_{i}=\left(2 I_{i} \omega_{i}^{\prime}\right)^{\frac{1}{2}} \cos \theta_{i} \quad(i=1,2), \\
& a_{i j}^{\prime}=a_{i j}\left(1+\alpha_{i j} \varepsilon+\alpha_{i j}^{\prime} \varepsilon^{\prime}\right), \quad i, j=1,2,3,4
\end{aligned}
$$


Restricting ourselves to only terms in $\varepsilon, \varepsilon^{\prime}$ and $\beta^{2}$ and neglecting their products, we find that the values of $a_{i j}$ are

$a_{11}=0, \quad a_{12}=0, \quad a_{13}=\frac{l_{1}}{2 k \omega_{1}}, \quad a_{14}=-\frac{l_{2}}{2 k \omega_{2}}$,

$a_{21}=-\frac{4 \omega_{1}}{k l_{1}}, \quad a_{22}=-\frac{4 \omega_{2}}{k l_{2}}, \quad a_{23}=-\frac{3 \sqrt{3} s}{2 k l_{1} \omega_{1}}$,

$a_{24}=\frac{3 \sqrt{3} s}{2 k l_{1} \omega_{1}}, \quad a_{31}=-\frac{m_{1} \omega_{1}}{2 k l_{1}}, \quad a_{32}=-\frac{m_{2} \omega_{2}}{2 k l_{2}}$,

$a_{33}=\frac{3 \sqrt{3} s}{2 k l_{1} \omega_{1}}, \quad a_{34}=-\frac{3 \sqrt{3} s}{2 k l_{2} \omega_{2}}, \quad a_{41}=\frac{3 \sqrt{3} s \omega_{1}}{2 k l_{1}}$,

$a_{42}=\frac{3 \sqrt{3} s \omega_{2}}{2 k l_{2}}, \quad a_{43}=\frac{n_{1}}{2 k l_{1} \omega_{1}}, \quad a_{44}=-\frac{n_{2}}{2 k l_{2} \omega_{2}}$

$k^{2}=\omega_{1}^{2}-\omega_{2}^{2}=2 \omega_{1}^{2}-1+\frac{3}{4} \beta^{2}=1-2 \omega_{2}^{2}-\frac{3}{4} \beta^{2}$

$(i=1,2)$

$l_{i}^{2}=4 \omega_{i}^{2}+9-\frac{77}{6} \beta^{2}, \quad m_{i}=4 \omega_{i}^{2}+1-\beta^{2}$,

$n_{i}=-4 \omega_{i}^{2}+9-\beta^{2} \quad(i=1,2)$

These values correspond to the classical problem with variable mass and for $\beta=0$, they agree with those found by Deprit and Deprit Bartholome (1967).

The values of $\alpha_{i j}$ and $\alpha_{i j}^{\prime}$ are exactly the same as worked out in the paper of Bhatnagar and Hallan (1978).

The transformation changes the second order part of the Hamiltonian into the normal form:

$\mathrm{H}_{2}=\omega_{1} I_{1}-\omega_{2} I_{2}$

The general solutions of the corresponding equations of motion are:

$I_{i}=$ const. $\quad(i=1,2)$

$\theta_{1}=\omega_{1}^{\prime} \Gamma+$ const. $\quad \theta_{2}=-\omega_{2}^{\prime} \Gamma+$ const.

\section{Second order normalization}

To perform Birkhoff's normalization we expand the coordinates in double d'Alembert series

$\xi=\sum_{n \geq 1} B_{n}^{1,0}, \quad \eta=\sum_{n \geq 1} B_{n}^{0,1}$

where the homogeneous components $B_{n}^{1,0}$ and $B_{n}^{0,1}$ of degree $n$ are of the form

$\sum_{0 \leq m \leq n} I_{1}^{(1 / 2)(n-m)} I_{2}^{\left(\frac{1}{2}\right) m} \sum_{(i, j)}\left[C_{n-m, m, i, j} \cos \left(i \theta_{1}+j \theta_{2}\right)\right.$
$\left.+S_{n-m, m, i, j} \sin \left(i \theta_{1}+j \theta_{2}\right)\right]$

The double summation over the indices $i$ and $j$ is such that (i) $i$ runs over those integers in the interval $0 \leq i \leq n-m$ that have the same parity as $n-m$, (ii) $j$ runs over those integers in the interval $-m \leq j \leq m$ that have the same parity as $m . I_{1}$ and $I_{2}$ are to be taken as constants of integration, while $\theta_{1}, \theta_{2}$ are to be determined as linear functions of time such that.

$\frac{d \theta_{1}}{d \Gamma}=\theta_{1}^{\prime}=\omega_{1}^{\prime}+\sum_{n \geq 1} f_{2 n}\left(I_{1}, I_{2}\right)$,

$\frac{d \theta_{2}}{d \Gamma}=\theta_{2}^{\prime}=-\omega_{2}^{\prime} \sum_{n \geq 1} g_{2 n}\left(I_{1}, I_{2}\right)$

where $f_{2 n}$ and $g_{2 n}$ are of the form

$$
\begin{aligned}
& f_{2 n}=\sum_{0 \leq m \leq n} f_{2(n-m), 2 m}^{\prime}, I_{1}^{n-m} I_{2}^{m}, \\
& g_{2 n}=\sum_{0 \leq m \leq n} g_{2(n-m), 2 m}^{\prime}
\end{aligned}
$$

The first order components $B_{1}^{1,0}$ and $B_{1}^{0,1}$ are the values of $\xi$ and $\eta$ given by (11). The second order components $B_{2}^{1,0}$ and $B_{2}^{0,1}$ are the solutions of the partial differential equations.

$\Delta_{1} \Delta_{2} B_{2}^{1,0}=\phi_{2}, \quad \Delta_{1} \Delta_{2} B_{2}^{0,1}=\psi_{2}$

where $\Delta_{i}=D^{2}+\omega_{i}^{2}(i=1,2)$

$$
\begin{aligned}
\phi_{2}= & {\left[D^{2}-3 B\left(1+\varepsilon^{\prime}\right)+\frac{1}{4} B^{\frac{5}{3}}\left(3+5 \varepsilon^{\prime}\right)\right] X_{2} } \\
& +\left[2(1+\varepsilon) D+\frac{s}{4} B^{\frac{5}{3}}\left(3+5 \varepsilon^{\prime}\right)\right. \\
& \left.\times\left\{4 B^{-\frac{2}{3}}\left(1-\frac{2}{3} \varepsilon^{\prime}\right)-1\right\}^{\frac{1}{2}}\right] Y_{2} \\
\psi_{2}= & {\left[D^{2}-\frac{1}{4} B^{\frac{5}{3}}\left(3+5 \varepsilon^{\prime}\right)\right] Y_{2} } \\
& -\left[2(1+\varepsilon)-\frac{s}{4} B^{\frac{5}{3}}\left(3+5 \varepsilon^{\prime}\right)\right. \\
& \left.\times\left\{4 B^{-\frac{2}{3}}\left(1-\frac{2}{3} \varepsilon^{\prime}\right)-1\right\}^{\frac{1}{2}}\right] X_{2},
\end{aligned}
$$

$D=\omega_{1}^{\prime}\left(\frac{\partial}{\partial \theta_{1}}\right)-\omega_{2}^{\prime}\left(\frac{\partial}{\partial \theta_{2}}\right)$ and $X_{2}, Y_{2}$ are obtained by substituting respectively in $\frac{\partial L_{3}}{\partial \xi}, \frac{\partial L_{3}}{\partial \eta}$ the first order components for $\xi$ and $\eta$. We can solve (12) for $B_{2}^{1,0}$ and $B_{2}^{0,1}$ by using the formula

$$
\frac{1}{\Delta_{1} \Delta_{2}}\left\{\begin{array}{l}
\cos \left(m \theta_{1}+n \theta_{2}\right) \\
\text { or } \\
\sin \left(m \theta_{1}+n \theta_{2}\right)
\end{array}\right\}=\frac{1}{\Delta_{m, n}}\left\{\begin{array}{l}
\cos \left(m \theta_{1}+n \theta_{2}\right) \\
\text { or } \\
\sin \left(m \theta_{1}+n \theta_{2}\right)
\end{array}\right\}
$$


where $\Delta_{m, n}=\left\lfloor\omega_{1}^{\prime 2}-\left(m \omega_{1}^{\prime}-n \omega_{2}\right)^{\prime 2}\right\rfloor\left\lfloor\omega_{2}^{\prime 2}-\left(m \omega_{1}^{\prime}-\right.\right.$ $\left.\left.n \omega_{2}^{\prime}\right)^{2}\right\rfloor$.

Provided $\Delta_{m, n} \neq 0$.

Since $\Delta_{1,0}=\Delta_{0,1}=0$, the terms $\cos \theta_{1}, \sin \theta_{1}, \cos \theta_{2}$, $\sin \theta_{2}$ are the critical terms. But $\phi_{2}$ and $\psi_{2}$ are free from such terms. By condition (i) of Moser's (1962) theorem, none of the divisors $\Delta_{2,0}, \Delta_{0,2} \Delta_{1,1}, \Delta_{1,-1}$ is zero. The second order components $B_{2}^{1,0}$ and $B_{2}^{0,1}$ are given as:

$$
\begin{aligned}
B_{2}^{1,0}= & r_{1}^{\prime} I_{1}+r_{2}^{\prime} I_{2}+r_{3}^{\prime} I_{1} \cos 2 \theta_{1}+r_{4}^{\prime} I_{2} \cos 2 \theta_{2} \\
& +r_{5}^{\prime} I_{1} \sin 2 \theta_{1}+r_{6}^{\prime} I_{2} \sin 2 \theta_{2}+r_{7}^{\prime} I_{1}^{\frac{1}{2}} I_{2}^{\frac{1}{2}} \cos \left(\theta_{1}+\theta_{2}\right) \\
& +r_{8}^{\prime} I_{1}^{\frac{1}{2}} I_{2}^{\frac{1}{2}} \cos \left(\theta_{1}+\theta_{2}\right)+r_{9}^{\prime} I_{1}^{\frac{1}{2}} I_{2}^{\frac{1}{2}} \sin \left(\theta_{1}+\theta_{2}\right) \\
& +r_{10}^{\prime} I_{1}^{\frac{1}{2}} I_{2}^{\frac{1}{2}} \sin \left(\theta_{1}-\theta_{2}\right)
\end{aligned}
$$

$B_{2}^{0,1}=s_{1}^{\prime} I_{1}+s_{2}^{\prime} I_{2}+s_{3}^{\prime} I_{1} \cos 2 \theta_{1}+s_{4}^{\prime} I_{2} \cos 2 \theta_{2}$

$$
\begin{aligned}
& +s_{5}^{\prime} I_{1} \sin 2 \theta_{1} \\
& +s_{6}^{\prime} I_{2} \sin 2 \theta_{2}+s_{7}^{\prime} I_{1}^{\frac{1}{2}} I_{2}^{\frac{1}{2}} \cos \left(\theta_{1}+\theta_{2}\right) \\
& +s_{8}^{\prime} I_{1}^{\frac{1}{2}} I_{2}^{\frac{1}{2}} \cos \left(\theta_{1}-\theta_{2}\right)+s_{9}^{\prime} I_{1}^{\frac{1}{2}} I_{2}^{\frac{1}{2}} \sin \left(\theta_{1}+\theta_{2}\right) \\
& +s_{10}^{\prime} I_{1}^{\frac{1}{2}} I_{2}^{\frac{1}{2}} \sin \left(\theta_{1}-\theta_{2}\right)
\end{aligned}
$$

where $r_{i}^{\prime}=r_{i}\left(1+\alpha_{i} \varepsilon+\alpha_{i}^{\prime} \varepsilon^{\prime}\right), s_{i}^{\prime}=s_{i}\left(1+\beta_{i} \varepsilon+\beta_{i}^{\prime} \varepsilon^{\prime}\right)$, $(i=1,2, \ldots, 10)$.

The values of all $r_{i}, s_{i}$ are:

$r_{1}=-\frac{3 s \gamma^{-\frac{1}{2}}}{8 \omega_{1} k^{2}}\left(11-\frac{4}{9} \beta^{2}\right)$

$$
\begin{aligned}
& r_{3}=-s \gamma^{-\frac{1}{2}}\left(\frac{-76 \omega_{1}^{4}+321 \omega_{1}^{2}+27+54 \beta_{1}^{2} \omega_{1}^{4}-214 \beta^{2} \omega_{1}^{2}-26 \beta^{2}-350 \beta^{2} \omega_{1}^{2}}{8 k^{2} l_{1}^{2} \omega_{1} z_{1}}\right), \\
& r_{5}=-\frac{\gamma^{-\frac{1}{2}}}{\sqrt{3} k^{2} l_{1}^{2} z_{1}}\left(44 \omega_{1}^{4}-53 \omega_{1}^{2}+18-14 \beta^{2} \omega_{1}^{4}+243 \beta^{2} \omega_{1}^{2}-32 \beta^{2}+135 \beta^{2} \omega_{1}^{-2}\right), \\
& r_{7}=\frac{35 \gamma^{-\frac{1}{2}}\left(-72 u^{2}+229 u-36+12 \beta^{2} u^{2}-192 \beta^{2} u-87 \beta^{2}-513 \beta^{2} u^{-1}\right)}{4 k^{2} l_{1} l_{2}\left(5 u-2+2 \beta^{2}\right) \sqrt{u}}, \\
& r_{9}=\sqrt{3} \gamma^{-\frac{1}{2}}\left(\omega_{1}-\omega_{2}\right)\left(\frac{-44 u^{2}+3 u+15+24 \beta^{2} u^{2}+50 \beta^{2} u+33 \beta^{2}-128 \beta^{2} u^{-1}}{k^{2} l_{1} l_{2}\left(5 u-2+2 \beta^{2}\right) \sqrt{u}}\right),
\end{aligned}
$$

$$
\begin{aligned}
& s_{1}=\frac{\sqrt{3} \gamma^{-\frac{1}{2}}}{24 \omega_{1} k^{2}}\left(8 \omega_{1}^{2}+9-243 \beta^{2}\right), \\
& s_{3}=-\frac{\sqrt{3} \gamma^{-\frac{1}{2}}}{72 k^{2} l_{1}^{2} \omega_{1} z_{1}}
\end{aligned}
$$

$$
\begin{aligned}
& \times\left(-736 \omega_{1}^{6}+1444 \omega_{1}^{4}-2733 \omega_{1}^{2}+729+308 \beta^{2} \omega_{1}^{6}\right. \\
& \left.+978 \beta^{2} \omega_{1}^{4}-2342 \beta^{2} \omega_{1}^{2}+3514 \beta^{2}+824 \beta^{2} \omega_{1}^{-2}\right), \\
s_{5}= & \frac{s \gamma^{-\frac{1}{2}}}{k^{2} l_{1}^{2} z_{1}}\left(-59 \omega_{1}^{2}+24-6 \beta^{2} \omega_{1}^{2}+77 \beta^{2}-87 \beta^{2} \omega_{1}^{-2}\right),
\end{aligned}
$$

$s_{7}=\frac{\sqrt{3} \gamma^{-\frac{1}{2}}\left(144 u^{3}-160 u^{2}+261 u-180-66 \beta^{2} u^{3}+2016 \beta^{2} u^{2}-2374 \beta^{2} u-1252 \beta^{2}+3469 \beta^{2} u^{-1}\right)}{12 k^{2} l_{1} l_{2}\left(5 u-2+2 \beta^{2}\right) \sqrt{u}}$,

$$
\begin{aligned}
s_{9}= & -3 s \gamma^{-\frac{1}{2}}\left(\omega_{1}-\omega_{2}\right) \\
& \times\left(\frac{7 u+9-704 \beta^{2} u+1161 \beta^{2}+4606 \beta^{2} u^{-1}}{k^{2} l_{1} l_{2}\left(5 u-2+2 \beta^{2}\right) \sqrt{u}}\right),
\end{aligned}
$$

$$
u=\omega_{1} \omega_{2}, \quad z_{i}=1-5 \omega_{i}^{2}-\beta^{2} \quad(i=1,2)
$$

The values of $r_{i}, s_{i}$ for $i=2,4,6$ can be obtained respectively from those for $i=1,3,5$ by replacing $\omega_{1}$ by $-\omega_{2}$, 
$l_{1}$ by $l_{2}, k^{2}$ by $-k^{2}, z_{1}$ by $z_{2}$ wherever they occur and the value of $r_{i}, s_{i}$, for $i=8,10$ can be obtained respectively from those for $i=7,9$ by replacing $\omega_{1}$ by $-\omega_{2}$ keeping $\omega_{1}$, $k^{2}, k^{4}, l_{1}, l_{1}^{2}, l_{2}, l_{2}^{2},\left(\omega_{1} \omega_{2}\right)^{1 / 2}$.

The values of $r_{i}, s_{i}$ correspond to the classical problem with variable mass and for $\beta=0, \gamma=1$, they agree with those obtained by Deprit and Deprit Bartholome (1967). The values of $\alpha_{i}, \alpha_{i}^{\prime}, \beta_{i}, \beta_{i}^{\prime}$ are the same as those given in the paper by Bhatnagar and Hallan (1978).

\section{Second order coefficients in the frequencies}

The third order components $B_{3}^{1,0}$ and $B_{3}^{0,1}$ in the coordinates $\xi, \eta$ and second order polynomials $f_{2}, g_{2}$ in the frequencies $\theta_{1}^{\prime}, \theta_{2}^{\prime}$ satisfy the partial differential equations

$$
\begin{aligned}
& \Delta_{1} \Delta_{2} B_{3}^{1,0}=\phi_{3}-2 f_{2} P-2 g_{2} Q, \\
& \Delta_{1} \Delta_{2} B_{3}^{0,1}=\psi_{3}-2 f_{2} U-2 g_{2} V
\end{aligned}
$$

where

$$
\begin{aligned}
& \phi_{3}= {\left[D^{2}-3 B\left(1+\varepsilon^{\prime}\right)+\frac{1}{4} B^{5 / 3}\left(3+5 \varepsilon^{\prime}\right)\right] X_{3} } \\
&+\left[2(1+\varepsilon) D+\frac{s}{4} B^{\frac{5}{3}}\left(3+5 \varepsilon^{\prime}\right)\right. \\
&\left.\times\left\{4 B^{-\frac{2}{3}}\left(1-\frac{2}{3} \beta \varepsilon^{\prime}\right)-1\right\}^{\frac{1}{2}}\right] Y_{3}, \\
& \psi_{3}= {\left[D^{2}-\frac{1}{4} B^{5 / 3}\left(3+5 \varepsilon^{\prime}\right)\right] Y_{3} } \\
&-\left[2(1+\varepsilon) D-\frac{s}{4} B^{5 / 3}\left(3+5 \varepsilon^{\prime}\right)\right. \\
&\left.\times\left\{4 B^{-\frac{2}{3}}\left(1-\frac{2}{3} \beta \varepsilon^{\prime}\right)-1\right\}^{\frac{1}{2}}\right] X_{3}, \\
& P= \frac{\partial}{\partial \theta_{1}}\left(\tau_{1} \tau_{2}+\tau_{3} \tau_{4}\right), \quad Q=\frac{\partial}{\partial \theta_{2}}\left(\tau_{5} \tau_{6}+\tau_{7} \tau_{8}\right), \\
& U=\frac{\partial}{\partial \theta_{i}}\left(\tau_{9} \tau_{10}-\tau_{11} \tau_{12}\right), \quad V=\frac{\partial}{\partial \theta_{2}}\left(\tau_{13} \tau_{14}+\tau_{15} \tau_{16}\right), \\
& \tau_{1}= \omega_{1}^{\prime} \frac{\partial^{2}}{\partial \theta_{1}^{2}}-\frac{1}{4} B^{\frac{5}{3}}\left[12 B^{-\frac{2}{3}}\left(1+\varepsilon^{\prime}\right)-\left(3+5 \varepsilon^{\prime}\right)\right], \\
& \tau_{2}= \omega_{1}^{\prime} \frac{\partial B_{1}^{1,0}}{\partial \theta_{1}}-(1+\varepsilon) B_{1}^{0,1}, \\
& \tau_{3}=2(1+\varepsilon) \omega_{1}^{\prime} \frac{\partial}{\partial \theta_{1}}+\frac{s}{4} B^{\frac{5}{3}}\left(3+5 \varepsilon^{\prime}\right) \\
& \times\left\{4 B^{-\frac{2}{3}}\left(1+\frac{2}{3} \varepsilon^{\prime}\right)-1\right\}^{\frac{1}{2}},
\end{aligned}
$$

$$
\begin{aligned}
& \tau_{4}= 2 \omega_{1}^{\prime} \frac{\partial B_{1}^{0,1}}{\partial \theta_{1}}+(1+\varepsilon) B_{1}^{1,0}, \\
& \tau_{5}= \omega_{2}^{\prime 2} \frac{\partial^{2}}{\partial \theta_{2}^{2}}-\frac{1}{4} B^{\frac{5}{3}}\left[12 B^{-\frac{2}{3}}\left(1+\varepsilon^{\prime}\right)-\left(3+5 \varepsilon^{\prime}\right)\right], \\
& \tau_{6}=-\omega_{2}^{\prime} \frac{\partial B_{1}^{1,0}}{\partial \theta_{2}}-(1+\varepsilon) B_{1}^{0,1}, \\
& \tau_{7}=-2(1+\varepsilon) \omega_{2}^{\prime} \frac{\partial}{\partial \theta_{2}} \\
&+\frac{s}{4} B^{\frac{5}{3}}\left(3+5 \varepsilon^{\prime}\right)\left\{4 B^{-\frac{2}{3}}\left(1-\frac{2}{3} \varepsilon^{\prime}\right)-1\right\}^{\frac{1}{2}}, \\
& \tau_{8}=-\omega_{2}^{1} \frac{d B_{1}^{0,1}}{d \theta_{2}}+(1+\varepsilon) B_{1,1}^{0,1}, \\
& \tau_{9}= \omega_{1}^{\prime 2} \frac{\partial^{2}}{\partial \theta_{1}^{2}}-\frac{1}{4} B^{\frac{5}{3}}\left(3+5 \varepsilon^{\prime}\right), \\
& \tau_{10}= \omega_{1}^{1} \frac{d B_{1}^{0,1}}{d \theta_{1}}+(1+\varepsilon) B_{1}^{1,0}, \\
& \tau_{11}= 2(1+\varepsilon) \omega_{1}^{\prime} \frac{\partial}{\partial \theta} \\
&-\frac{s}{4} B_{2}^{\prime} \frac{\partial B_{1}^{1,0}}{\partial \theta_{2}}+(1+\varepsilon) B_{1}^{0,1}, \\
& \tau_{14}=-\omega_{2}^{\prime} \frac{\partial B_{1}^{0,1}}{\partial \theta_{2}}+\left(3+5 \varepsilon^{\prime}\right)\left\{4 B^{-\frac{2}{3}}\left(1-\frac{2}{3} \varepsilon^{\prime}\right)-1\right\}^{\frac{1}{2}}, \\
& \tau_{12}=-2(1+\varepsilon) \omega_{1}^{\prime} \frac{\partial B_{1}^{1,0}}{\partial \theta_{1}}-(1+\varepsilon) B_{2}^{\prime 2} \frac{\partial^{2}}{\partial \theta_{2}^{2}}-\frac{1}{4} B^{\frac{5}{3}}\left(3+5 \varepsilon^{\prime}\right), \\
& 0,1
\end{aligned}
$$

and $X_{3}, Y_{3}$ are the homogeneous components of order 3 obtained by substituting respectively in

$$
\begin{aligned}
& \left(\frac{\partial}{\partial \xi}\right)\left(\mathrm{L}_{3}+\mathrm{L}_{4}\right),\left(\frac{\partial}{\partial \eta}\right)\left(\mathrm{L}_{3}+\mathrm{L}_{4}\right), \quad \xi=B_{1}^{1,0}+B_{2}^{1,0}, \\
& \eta=B_{1}^{0,1}+B_{2}^{0,1}
\end{aligned}
$$

We do not require to find out the components $B_{3}^{1,0}$ and $B_{3}^{0,1}$. We only find the coefficients of $\cos \theta_{1}, \sin \theta_{1}, \cos \theta_{2}$ and $\sin \theta_{2}$ in the right hand sides of (13). They are the critical terms. We eliminate these terms by choosing properly the 
coefficients in the polynomials

$f_{2}=f_{2,0}^{\prime} I_{1}+f_{0,2}^{\prime} I_{2,} g_{2}=g_{2,0}^{\prime} I_{1}+g_{0,2}^{\prime} I_{2}$

We find that

$$
\begin{aligned}
& A=f_{2,0}^{\prime}=f_{2,0}^{\prime}\left[1+\left(\varsigma_{1}-\varsigma_{3}\right) \varepsilon+\left(\varsigma_{1}^{\prime}-\varsigma_{3}^{\prime}\right) \varepsilon^{\prime}\right], \\
& \bar{B}=f_{2,0}^{\prime}=g_{2,0}^{\prime}=f_{0,2}\left[1+\left(\varsigma_{2}-\varsigma_{3}\right) \varepsilon+\left(\varsigma_{2}^{\prime}-\varsigma_{3}^{\prime}\right) \varepsilon^{\prime}\right], \\
& C=g_{0,2}^{\prime}=g_{0,2}\left[1+\left(\varsigma_{4}-\varsigma_{5}\right) \varepsilon+\left(\varsigma_{4}^{\prime}-\varsigma_{5}^{1}\right) \varepsilon^{\prime}\right]
\end{aligned}
$$

The values of $f_{2,0}, f_{0,2}, g_{0,2}$ are

$$
\begin{aligned}
f_{2,0}= & \omega_{2}^{2} \gamma^{-1}\left[124 \omega_{1}^{4}-696 \omega_{1}^{2}+81-42922 \beta^{2} \omega_{1}^{4}\right. \\
& \left.+107473 \beta^{2} \omega_{1}^{2}-720306 \beta^{2}\right] \\
& /\left[72\left(1-2 \omega_{1}^{2}-\frac{3}{4} \beta^{2}\right)\left(1-5 \omega_{1}^{2}-\beta^{2}\right)\right], \\
f_{0,2}= & -\omega_{1} \omega_{2} \gamma^{-1}\left[64 \omega_{1}^{2} \omega_{2}^{2}+43+142674 \beta^{2} \omega_{1}^{2} \omega_{2}^{2}\right. \\
& \left.-2219166 \beta^{2}\right] \\
& /\left[6\left(1-2 \omega_{1}^{2}-\frac{3}{4} \beta^{2}\right)\left(1-2 \omega_{2}^{2}-\frac{3}{4} \beta^{2}\right)\right. \\
& \left.\times\left(1-5 \omega_{1}^{2}-\beta^{2}\right)\right], \\
g_{0,2}= & \omega_{1}^{2} \gamma^{-1}\left[124 \omega_{2}^{4}-696 \omega_{2}^{2}+81-42922 \beta^{2} \omega_{2}^{4}\right. \\
& \left.+107473 \beta^{2} \omega_{2}^{2}-720306 \beta^{2}\right] \\
& /\left[72\left(1-2 \omega_{2}^{2}-\frac{3}{4} \beta^{2}\right)\left(1-5 \omega_{2}^{2}-\beta^{2}\right)\right]
\end{aligned}
$$

These values correspond to those of the classical problem with variable mass and if $\gamma=1, \beta=0$, they agree with those found by Deprit and Deprit Bartholome (1967).

The coefficients of $\varepsilon$ and $\varepsilon^{\prime}$ are the same as worked out in the paper of Bhatnagar and Hallan (1978).

\section{Stability}

For complete investigation of the problem of stability of $\mathrm{L}_{4}$ we must consider the cases in which the conditions (i) and (ii) of Moser's (1962) theorem are not satisfied. In calculating $B_{2}^{1,0}, B_{2}^{0,1}, B_{3}^{1,0}, B_{3}^{0,1}$ we have assumed the condition (i). Now we wish to verify that this condition is satisfied. The condition is $k_{1} \omega_{1}^{\prime}+k_{2} \omega_{2}^{\prime} \neq 0$ for all pairs of integers $\left(k_{1}, k_{2}\right)$ such that $\left|k_{1}\right|+\left|k_{2}\right| \leq 4$.

Now,

$k_{1} \omega_{1}^{\prime}+k_{2} \omega_{2}^{\prime}=0 \Leftrightarrow \frac{\omega_{1}^{\prime}}{\omega_{2}^{\prime}}=-\frac{k_{2}}{k_{1}}$

Also, (8) implies that $\frac{\omega_{1}^{\prime}}{\omega_{2}^{\prime}}>1$.
For (14) to be true, $k_{1}$ and $k_{2}$ are of opposite signs and $\frac{-k_{2}}{k_{1}}>1$. This arises two cases: $\frac{\omega_{1}^{\prime}}{\omega_{2}^{\prime}}=2$ and $\frac{\omega_{1}^{\prime}}{\omega_{2}^{\prime}}=3$. From $\frac{\omega_{1}^{\prime}}{\omega_{2}^{\prime}}=2$ and first of equation (8) we have

$\omega_{1}^{\prime}=\frac{2}{\sqrt{5}}\left[1+4 \varepsilon-\frac{3}{2} \varepsilon^{\prime}-\frac{3}{8} \beta^{2}\right]$,

$\omega_{2}^{\prime}=\frac{1}{\sqrt{5}}\left[1+4 \varepsilon-\frac{3}{2} \varepsilon^{\prime}-\frac{3}{8} \beta^{2}\right]$

Putting these values in the second equation of (8) with $s=$ $1-2 \mu$ and solving for $\mu$, we find that (14) is true for $\mu=$ $\mu_{1}^{\prime}$. Proceeding as above for the case $\frac{\omega_{1}^{\prime}}{\omega_{2}^{\prime}}=3$, we see that (14) is true for $\mu=\mu_{2}^{\prime}$,

Thus, condition (i) of Moser's (1962) theorem is satisfied in the interval $0<\mu<\mu_{\mathrm{c}}$ if the mass ratio does not take the critical values:

$\mu_{i}^{\prime}=\mu_{1}+\frac{64\left(36 \varepsilon-19 \varepsilon^{\prime}\right)}{135 \sqrt{1833}}, \quad \mu_{2}^{\prime}=\mu_{2}+\frac{4\left(36 \varepsilon-19 \varepsilon^{\prime}\right)}{45 \sqrt{213}}$

where

$$
\begin{aligned}
\mu_{i} & =\frac{1}{2}\left[\left(1-\frac{\sqrt{1833}}{45}\right)-\frac{608}{135 \sqrt{1833}} \beta^{2} r\right] \\
& =0.02429389 \ldots-\frac{304}{135 \sqrt{1833}} \beta^{2}, \\
\mu_{2} & =\frac{1}{2}\left[\left(1-\frac{\sqrt{213}}{15}\right)-\frac{38}{45 \sqrt{213}} \beta^{2}\right] \\
& =0.01351601 \ldots-\frac{19}{45 \sqrt{213}} \beta^{2}
\end{aligned}
$$

The critical values $\mu_{1}^{\prime}$ and $\mu_{2}^{\prime}$ correspond to the resonance cases $1: 2$ and 1:3.

The normalized Hamiltonian up to fourth order is

$\mathrm{H}=\omega_{1}^{\prime} I_{1}-\omega_{2}^{\prime} I_{2}+\frac{1}{2}\left(A I_{1}^{2}+2 \bar{B} I_{1} I_{2}+C I_{2}^{2}\right)$.

The determinant $D$ occurring in condition (ii) of Moser's (1962) theorem is

$$
\begin{aligned}
D= & -\gamma^{-1}\left[9 \left(644 u^{4}-541 u^{2}+36\right.\right. \\
& \left.\left.-\left(540725 u^{4}-7157934 u^{2}+320243\right) \beta^{2}+R \varepsilon+R^{\prime} \varepsilon^{\prime}\right)\right] \\
& / 72\left(-4 U^{2}+1-\frac{3}{2} \beta^{2}\right)\left(-25 U^{2}+4-\frac{27}{4} \beta^{2}\right)
\end{aligned}
$$

where $R$ and $R^{\prime}$ are the same as those obtained in the paper of Bhatnagar and Hallan (1978). Condition (ii) of Moser's (1962) theorem is satisfied i.e. $D \neq 0$ if in the interval $0<$ $\mu<\mu_{\mathrm{c}}$, mass ratio does not take the value

$\mu_{3}^{\prime}=\mu_{3}\left(1+Y \varepsilon+Y^{\prime} \varepsilon^{\prime}\right)$, 


$$
\begin{aligned}
\mu_{3}= & \frac{1}{2}\left[1-\left\{\left(27 b_{5}-16 b_{1}+16 \sqrt{b_{3}}\right) / 27 b_{5}\right\}^{\frac{1}{2}}\right] \\
& +\left[2\left\{2 b_{6}\left(b_{1}-\sqrt{b_{3}}\right) / b_{5}-b_{4} / \sqrt{b_{3}}-2 b_{2}\right\}\right. \\
& \left./\left(27 b_{5}\right)^{\frac{1}{2}}\left(27 b_{5}-16 b_{1}+16 \sqrt{b_{3}}\right)^{\frac{1}{2}}\right] \beta^{2}, \\
b_{1}= & 541, b_{2}=7157934, b_{3}=199945, b_{4}=1444952726, \\
b_{5}= & 1288, b_{6}=1080663, \gamma=250.922 \text { and } \gamma^{\prime}=-166.304, \\
\mu_{3}= & 0.010913668 \ldots-181.9898854 \ldots \beta^{2}
\end{aligned}
$$

In the computation of $\mu_{i}^{\prime}(i=1,2,3)$ we have retained terms up to $\beta^{2}, \varepsilon, \varepsilon^{\prime \prime}$ and have neglected their products.

The values of $\mu_{I}(i=1,2,3)$ correspond to those of the restricted three-body problem with variable mass and if $\beta=0$, they agree with those found by Deprit and Deprit Bartholome (1967). If $\beta=0, \mu_{i}^{\prime}$ reduces to the results obtained by Bhatnagar and Hallan (1978).

\section{Conclusion}

By taking perturbations $\varepsilon$ and $\varepsilon^{\prime}$ in the Coriolis and the centrifugal forces respectively, we have found that in the nonlinear sense, triangular points are stable in the range of linear stability except for three mass ratios:

$$
\begin{aligned}
\mu_{i}^{\prime}= & 0.02429389 \ldots-\frac{304 \beta^{2}}{135 \sqrt{1833}}+\frac{64\left(36 \varepsilon-19 \varepsilon^{\prime}\right)}{135 \sqrt{1833}}, \\
\mu_{2}^{\prime}= & 0.01351601 \ldots-\frac{19 \beta^{2}}{45 \sqrt{213}}+\frac{4\left(36 \varepsilon-19 \varepsilon^{\prime}\right)}{45 \sqrt{213}}, \\
\mu_{3}^{\prime}= & \left(0.01091368 \ldots-181.9898854 \ldots \beta^{2}\right) \\
& \times\left(1+250.922 \varepsilon-166.304 \varepsilon^{\prime}\right)
\end{aligned}
$$

at which Moser's (1962) theorem is not applicable.

When $\beta=\varepsilon=\varepsilon^{\prime}=0$, the values of $\mu_{i}^{\prime}(i=1,2,3)$ are in agreement with those of Deprit and Deprit Bartholome (1967). When $\beta=0$, the results obtained correspond to Bhatnagar and Hallan (1978). When $\varepsilon=\varepsilon^{\prime}=0$, the case corresponds to the classical problem with variable mass.
Acknowledgements The author is extremely thankful to Professor B. Ishwar, Department of Mathematics, B.R.A. Bihar University, Muzaffarpur, India for his valuable suggestions.

Open Access This article is distributed under the terms of the Creative Commons Attribution Noncommercial License which permits any noncommercial use, distribution, and reproduction in any medium, provided the original author(s) and source are credited.

\section{References}

AbdulRaheem, A., Singh, J.: Astron. J. 131, 1880 (2006)

Arnold, V.I.: Soviet Math. Dokl. 2, 247 (1961)

Bekov, A.A.: Instability, Chaos and Predictability in Celestial Mechanics and Stellar Dynamics, p. 277. Nova Science Publishers, New York (1992)

Bhatnagar, K.B., Hallan, P.P.: Celest. Mech. 18, 105 (1978)

Contopoulos, G.: Order and Chaos in Dynamical Astronomy, p. 543. Springer, Berlin (2002)

Deprit, A., Deprit Bartholome, A.: Astron. J. 72, 173 (1967)

Gozdziewski, K.: Celest. Mech. Dyn. Astron. 85, 79 (2003)

Hallan, P.P., Jain, S., Bhatnagar, K.B.: Celest. Mech. Dyn. Astron. 77, 157 (2000)

Ishwar, B.: Celest Mech. Dyn. Astron. 65, 253 (1997)

Jeans, J.H.: Astronomy and Cosmogony. Cambridge University Press, London (1928)

Liapunov, A.M.: A General Problem of Stability of Motion. Acad. Sci. USSR, Moscow (1956)

Meshcherskii, I.V.: Studies on the Mechanics of Bodies of Variable Mass. Moscow, Leningrad (1949)

Moser, J.K.: Nachr. Akad. Wiss. Gottingen, Math-Phys. KI, 1 (1962)

Niedzielska, Z.: Celest. Mech. Dyn. Aston. 58, 203 (1994)

Shrivastava, A.K., Ishwar, B.: Celest. Mech. 30, 323 (1983)

Singh, J., Ishwar, B.: Celest. Mech. 32, 297 (1984)

Singh, J., Ishwar, B.: Celest. Mech. 35, 201 (1985)

Subbarao, P.V., Sharma, R.K.: Astron. Astrophys. 43, 381 (1975)

Subbarao, P.V., Sharma, R.K.: Celest. Mech. Dyn. Astron. 65, 291 (1997)

Szebehely, V.: Theory of Orbits: The Restricted Problem of Three Bodies, pp. 244-264. Academic Press, New York (1967a)

Szebehely, V.: Astron. J. 72, 7 (1967b)

Verhulst, F.: Celest. Mech. 5, 27 (1972)

Whittaker, E.T.: A Treatise on the Analytical Dynamics of Particles and Rigid Bodies, pp. 427-430. Cambridge University Press, London (1965)

Wintner, A.: The Analytical Foundations of Celestial Mechanics, pp. 372-373. Princeton University Press, Princeton (1941) 